\title{
Pinturas alquídicas con mordenita intercambiada con La (III) y Pr (III) como pigmento anticorrosivo para la protección de acero SAE 1010
}

\author{
Alkyd paints with mordenite exchanged with La (III) and $\operatorname{Pr}($ III) \\ as anticorrosive pigment for SAE1010 steel protection \\ Oriana D’Alessandro ${ }^{1 *} \quad$ Gonzalo Selmi $^{2} \quad$ Cecila Deyá $^{3} \quad$ Roberto Romagnoli ${ }^{2}$ \\ Recibido 14 de septiembre de 2018, aceptado 25 de marzo de 2019 \\ Received: September 14, 2018 Accepted: March 25, 2019
}

\begin{abstract}
RESUMEN
El presente trabajo tuvo como objetivo la formulación y elaboración de pinturas alquídicas con nuevos pigmentos anticorrosivos a base de mordenita intercambiada, amigables con el medio ambiente. La caracterización electroquímica del acero pintado se llevó a cabo por: medidas de conductividad, ensayos de polarización lineal y medidas del potencial de corrosión. La película de pintura ensayada en cada caso fue removida y la superficie fue analizada mediante microscopía electrónica de barrido y análisis de energía dispersiva por rayos $\mathrm{X}$.

Los resultados muestran que los nuevos pigmentos incorporados a la pintura proporcionan propiedades anticorrosivas aceptables teniendo en cuenta su bajo impacto ambiental y la utilización de un recurso natural de bajo costo.
\end{abstract}

Palabras clave: Mordenita, lantano, praseodimio, pintura alquídica.

\section{ABSTRACT}

The objective of this work was to formulate and prepare alkyd paints with new anticorrosive pigments based on exchanged mordenite, friendly with the environment. The electrochemical characterization of painted steel was done by: conductivity measurements, linear polarizations essays and corrosion potential measurements. The paint film tested in this case was removed and the steel surface analysed by scanning electron microscopy and energy dispersive $X$-ray analysis.

The results showed that the new pigments incorporated in the paint have acceptable anticorrosive properties, taking into account their low environmental impact and the use of a low-cost natural resource.

Keywords: Mordenite, lanthanum, praseodymium, alkyd paint.

\section{INTRODUCCIÓN}

La corrosión es el deterioro de los materiales en el medio en el que son utilizados. En el caso de los aceros se puede hablar de un proceso electroquímico que ocurre en la interface metal-medio agresivo. Una de las estrategias ampliamente utilizadas para la protección de superficies metálicas es la aplicación de pinturas anticorrosivas. La protección se logra a través de dos efectos: el "efecto barrera" donde la

\footnotetext{
1 Universidad Nacional de La Plata. Facultad de Ciencias Exactas. La Plata, Argentina. E-mail: o.dalessandro@cidepint.ing.unlp.edu.ar

2 CIDEPINT (CICPBA-CONICET-UNLP Facultad de Ingeniería). La Plata, Argentina.

E-mail: g.selmi@cidepint.gov.ar; direccion@cidepint.gov.ar

3 Universidad Nacional de La Plata. Facultad de Ingeniería. La Plata, Argentina. E-mail: c.deya@cidepint.gov.ar

* Autor de correspondencia: o.dalessandro@cidepint.ing.unlp.edu.ar
} 
película retarda la penetración de los agentes agresivos (agua, oxígeno y electrolitos) y el "efecto inhibidor" el cual está asociado a la incorporación de pigmentos anticorrosivos adecuados. Los pigmentos anticorrosivos tradicionales contenían $\mathrm{Pb}_{3} \mathrm{O}_{4}$ o $\mathrm{CrO}_{4}{ }^{2-}$, los cuales desde hace años han sido ampliamente cuestionados por su toxicidad. Entre los reemplazos, los fosfatos inorgánicos de $1^{\text {era }}, 2^{\text {da }}$ y $3^{\text {era }}$ generación fueron muy utilizados, hasta que estudios medioambientales revelaron el empobrecimiento de la biodiversidad por aumento de la biomasa y la consiguiente eutrofización de los ecosistemas acuáticos [1].

En la actualidad se cuenta con una gran variedad de pigmentos inhibidores alternativos, algunos de los cuales pueden emplearse como reservorios para cationes y/o aniones inhibidores. El mecanismo de acción de los inhibidores catiónicos parece estar relacionado con modificaciones cinéticas de la reducción catódica de oxígeno [2].

Las zeolitas son aluminosilicatos, cuya estructura consiste en un entramado tridimensional de tetraedros $\mathrm{SiO}_{4}{ }^{4-}$ con algunas sustituciones de $\mathrm{Si}$ por $\mathrm{Al}$, las cuales introducen una carga negativa que debe equilibrarse con un catión.

Estos materiales son considerados microporosos según la convención IUPAC, porque el tamaño medio de poro es menor a $2 \mathrm{~nm}$ y pueden clasificarse según el número de tetraedros que formen el anillo de mayor abertura.

La Mordenita es una zeolita caracterizada por presentar anillos de 12 y de 8 tetraedros [3] y el código para nombrarlas, utilizado por la International Mineralogical Association, es MOR $\left(\left(\mathrm{Na}_{2}, \mathrm{Ca}_{4}, \mathrm{~K}_{2}\right)_{4}\left[\mathrm{Al}_{8} \mathrm{Si}_{40} \mathrm{O}_{96}\right] * 28 \mathrm{H}_{2} \mathrm{O}\right)$ [4]. Los anillos de 12 miembros miden $0,65 \times 0,70 \mathrm{~nm}$ y los de 8 miembros miden $0,26 \times 0,57 \mathrm{~nm}$ [5].

La MOR natural ha sido estudiada como adsorbente para la remoción de varios contaminantes como $\mathrm{Hg}$ (II) [6] y Cr (III) [7] en aguas de desecho.

Uno de los usos de la forma sódica, Na-MOR, es como adsorbente de $\mathrm{Ni}$ (II) en aguas residuales [8] $y$ de $\mathrm{CO}_{2}$ en corrientes de $\mathrm{N}_{2}$ [9].

Cuando el catión equilibrante es un protón el material puede denominarse H-MOR, y presenta las propiedades de un ácido por lo cual ha sido ampliamente utilizado como un catalizador ácido en la síntesis de metilaminas a partir de metanol y amoníaco [10], en la conversión de metanol a dimetiléter [11] y en la esterificación de etanol y glicerol [12] entre otras.

Por su capacidad de alojar diferentes cationes, las zeolitas han sido estudiadas recientemente como contenedores de cationes pasivantes. La clinoptilolita intercambiada con La (III) [13] y una mezcla 50/80\% mordenita/heulandita intercambiada con Ce (III), han sido reportadas como pigmentos anticorrosivos en varias pinturas [14].

El objetivo de este trabajo de investigación fue estudiar el comportamiento anticorrosivo de dos pinturas alquídicas formuladas con MOR intercambiada con Pr (III) en un caso y con La (III) en otro, aplicadas sobre acero SAE 1010. La efectividad de estos recubrimientos se evaluó mediante la determinación de la resistencia iónica (como una propiedad inversamente proporcional a la conductividad), por ensayos de polarización lineal y por medida de potencial de corrosión. La variación de los parámetros proporcionados por estos ensayos en función del tiempo de inmersión en un medio agresivo, brinda información acerca del proceso de deterioro de la barrera protectora y de la acción anticorrosiva de los pigmentos estudiados.

\section{MATERIALES Y MÉTODOS}

\section{Formulación, preparación y aplicación de las pinturas anticorrosivas}

Las pinturas anticorrosivas se prepararon teniendo en cuenta una formulación típica [15] reemplazando, en volumen, el pigmento ACTIROX 102 HISPANIA ("molibdofosfato" de zinc, ZMP) por los pigmentos Mordenita intercambiada con Lantano (La-MOR) y Mordenita intercambiada con Praseodimio (Pr-MOR).

Los pigmentos zeolíticos fueron preparados en el laboratorio a partir de una zeolita natural, la cual mediante el análisis por difracción de rayos $\mathrm{X}$ y utilizando la base de datos de Join Committee for Powder Diffraction Sources fue identificada como Mordenita (JCPDF\# 22-1339). Por espectroscopía infrarroja con transformada de Fourier se observaron las bandas asociadas a las vibraciones internas de los tetraedros a $760 \mathrm{~cm}^{-1}$ y a las vibraciones entre tetraedros 
Tabla 1. Composición de la pintura anticorrosiva.

\begin{tabular}{|l|l|c|}
\hline \multicolumn{1}{|c|}{ Componentes } & \multicolumn{1}{c|}{ Función } & $\begin{array}{c}\text { \% en } \\
\text { volumen }\end{array}$ \\
\hline Pigmento principal & Pigmento anticorrosivo & 6,54 \\
\hline Talco & Carga & 6,37 \\
\hline Barita & Extendedor & 6,20 \\
\hline $\mathrm{TiO}_{2}$ & Opacante & 2,55 \\
\hline ALKIPOL $\AA 434 / 50$ & Resina alquídica & 60,23 \\
\hline Aguarrás & Solvente & 18,11 \\
\hline Total & & 100,00 \\
\hline
\end{tabular}

a $840 \mathrm{~cm}^{-1}$ típicas de la Mordenita. El intercambio se realizó con La (III) en un caso y con Pr (III) en otro, estos cationes fueron seleccionados luego del estudio de su capacidad como inhibidores de corrosión en solución a diferentes concentraciones [16].

Los componentes de las pinturas se pesaron con una precisión de $0,1 \mathrm{~g}$ y las pinturas se prepararon en un molino de bolas Hulman Hnos. Srl. a 70 rpm durante 24 horas.

La preparación de la superficie se llevó a cabo mediante arenado del sustrato metálico hasta alcanzar una rugosidad total de $25 \pm 2 \mu \mathrm{m}$ determinada con un Rugosímetro Hommel Tester LV15 y posteriormente se desengrasaron los paneles con tolueno.

La aplicación de las pinturas se realizó con pincel. El espesor total de la película seca obtenido luego de dos aplicaciones, con un tiempo de curado de 48 horas, fue de $75 \pm 5 \mu \mathrm{m}$, determinado con el medidor de espesores Schwyz SC117-02 Coating Thickness Gauge.

\section{Caracterización electroquímica del recubrimiento}

Los paneles pintados se caracterizaron electroquímicamente por medidas de conductividad para determinar la resistencia iónica (Ri), por ensayos de polarización lineal, una vez que ha permeado el agua, para determinar la resistencia a la polarización (Rp) y por medidas del potencial de corrosión (Ecorr).

Los ensayos electroquímicos se realizaron en una serie de celdas cuyo esquema se observa en la Figura 1. Las mismas fueron construidas delimitando sobre la superficie pintada un área circular de $3 \mathrm{~cm}^{2}$ mediante un tubo de policloruro de vinilo (PVC). Cada tubo se adhiere con pegamento de tipo epoxídico y luego se sella con cera de abeja por el exterior

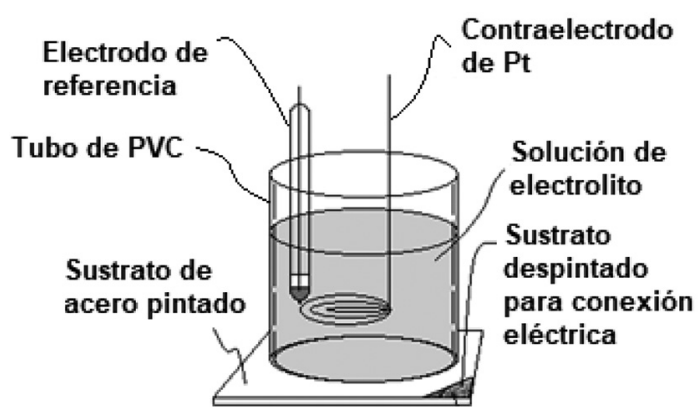

Figura 1. Celda electroquímica.

del mismo. Finalmente se adiciona un volumen definido de $\mathrm{NaCl}$ 0,1 M como electrolito soporte. La resistencia entre el sustrato de acero pintado y un electrodo de platino se determinó utilizando un conductímetro ATI Orion Model 170 a 1000 Hz. Los ensayos de polarización lineal se realizaron con un potenciostato Gamry Interface 1000. El rango de barrido fue $\pm 20 \mathrm{mV}$, y la velocidad de barrido $1 \mathrm{mV} / \mathrm{s}$ respecto al potencial a circuito abierto. Como contraelectrodo se utilizó un aro de Pt y el electrodo de referencia fue el de calomel saturado (ECS). Para la medida de potencial de corrosión también se utilizó el ECS como referencia. Todas las medidas se realizaron por cuadruplicado [17].

\section{Análisis de la superficie}

Los ensayos electroquímicos se extendieron a lo largo de 67 días. Luego, las celdas se enjuagaron con agua destilada 3 veces y los tubos de PVC fueron retirados. Se intentó remover la película de pintura por métodos mecánicos y al no conseguirlo, se optó por el método químico, es decir, se sumergieron las muestras pintadas en una solución de $\mathrm{NaOH}$ al $10 \% \mathrm{p} / \mathrm{v}$ durante 7 horas para romper los enlaces ésteres presentes en la resina alquídica.

Finalmente, las superficies fueron analizadas mediante microscopio electrónico de barrido (MEB) y análisis de energía dispersiva por rayos X (AED), previo metalizado con oro, para así determinar las características morfológicas y la composición final de la película formada sobre el sustrato.

\section{RESULTADOS Y DISCUSIÓN}

Caracterización electroquímica del recubrimiento La resistencia iónica (Ri) del esquema de pintado, aporta información sobre la penetración del agua 
y del electrolito a través de la película, en función del tiempo. Inicialmente se observó una elevada resistencia iónica, lo cual indica que la pintura actuó prácticamente como un dieléctrico hasta que, a partir de un determinado tiempo, la película permitió el paso del agua a través de ella. Se considera una buena protección cuando la resistencia del recubrimiento supera los $10^{8} \Omega . \mathrm{cm}^{2}$, mientras que una protección es deficiente cuando la resistencia del recubrimiento resulta inferior a $10^{6} \Omega . \mathrm{cm}^{2}[18]$.

En la Figura 2 se presentan las curvas de Ri en función del tiempo. Para todas las muestras se observaron valores iniciales de Ri menores a $10^{8} \Omega . \mathrm{cm}^{2}$, lo cual estaría indicando que las propiedades de barrera iniciales de las películas de pintura no eran las mejores. La pintura ZMP es la que presentó el mejor comportamiento, el valor Ri inicial fue mayor que para las otras dos pinturas y la caída de Ri a valores alrededor de $10^{3} \Omega . \mathrm{cm}^{2}$, escaso efecto barrera, se alcanzó luego de 40 días de ensayo. Las muestras La-MOR y Pr-MOR alcanzaron valores similares luego de 10 y 20 días de ensayo, respectivamente.

Cuando el efecto barrera disminuye resulta posible la determinación de los valores de resistencia a la polarización.

La resistencia a la polarización $(\mathrm{Rp})$ permite evaluar la velocidad de corrosión del sustrato pintado una vez que el agua ha alcanzado la superficie metálica. Los valores de referencia utilizados para el análisis son los que ya se han considerado anteriormente.

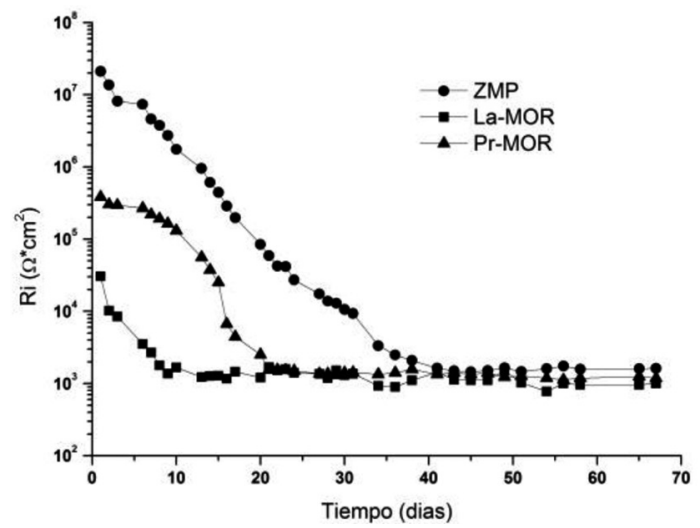

Figura 2. Resistencia iónica (Ri) en función del tiempo.
Según los datos presentados en la Figura 3, las medidas de polarización lineal pudieron realizarse luego de un día de comenzado el ensayo para los recubrimientos La-MOR y Pr-MOR. Esto estaría indicando una mejor protección del sustrato por la pintura ZMP, es decir, el proceso de corrosión no se detectó hasta después de las dos semanas de inmersión, debido a que prevaleció el efecto barrera de la película de pintura, $\mathrm{Ri}>10^{6} \Omega \cdot \mathrm{cm}^{2}$ (ver Figura 1).

Las tres muestras presentaron un comportamiento similar, la resistencia a la polarización tomó un valor inicial que cayó monótonamente con el tiempo hasta alcanzar un valor asintótico de alrededor de $4,510^{3} \Omega . \mathrm{cm}^{2}$ para el cual no existe protección anticorrosiva del sustrato.

Es de destacar que la pintura conteniendo Pr-MOR tuvo durante los primeros 4 días una buena resistencia a la polarización, o baja velocidad de corrosión, que luego cayó con el tiempo.

Las medidas de potencial de corrosión contra un electrodo de calomel saturado (ECS), en función del tiempo, permitieron monitorear el estado de la superficie metálica y los cambios del estado pasivo al estado activo. Además, permitieron tener una apreciación del tiempo a partir del cual comenzó el proceso de corrosión electroquímica.

En la Figura 4 se presentan las curvas de Ecorr vs ECS en función del tiempo de ensayo.

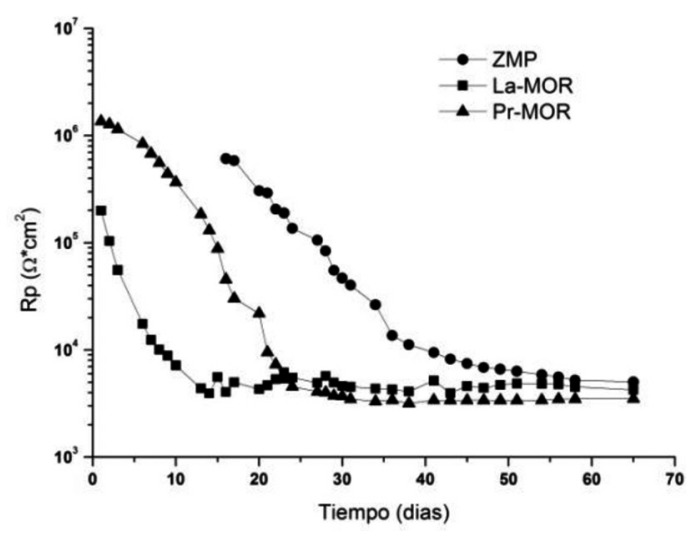

Figura 3. Resistencia a la polarización (Rp) en función del tiempo. 


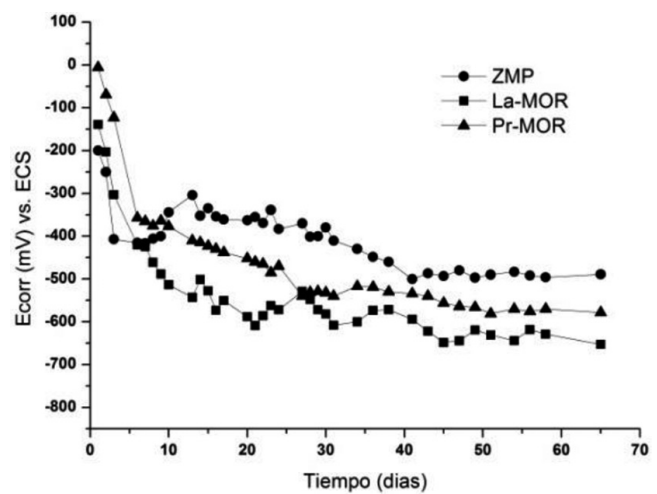

Figura 4. Potencial de corrosión (Ecorr) vs. ECS en función del tiempo.

Los valores iniciales de Ecorr para las tres muestras indicaron que el sustrato de acero está pasivado. Luego para todas las muestras se observó que el potencial de corrosión cayó monótonamente durante 45 días de ensayo, aunque con pequeñas oscilaciones debido a procesos de corrosiónformación de óxidos-tapado de poros-corrosión. A este tiempo la muestra La-MOR alcanzó el potencial de corrosión del acero desnudo de $-650 \mathrm{mV}$, mientras que la muestra Pr-MOR y ZMP alcanzaron los valores -560 y $-495 \mathrm{mV}$ respectivamente, luego los valores de potencial se mantuvieron en un valor constante hasta el final del ensayo.

\section{Análisis de la superficie}

Los electrodos de trabajo utilizados para los ensayos electroquímicos se observaron mediante MEB con la finalidad de caracterizar las diferentes superficies en su estado final y la composición de la película protectora fue determinada por AED. En la Figura 5 se presentan las microfotografías (500x) de los electrodos de trabajo una vez removida la película de pintura al finalizar el ensayo electroquímico y en la Tabla 2 se muestran
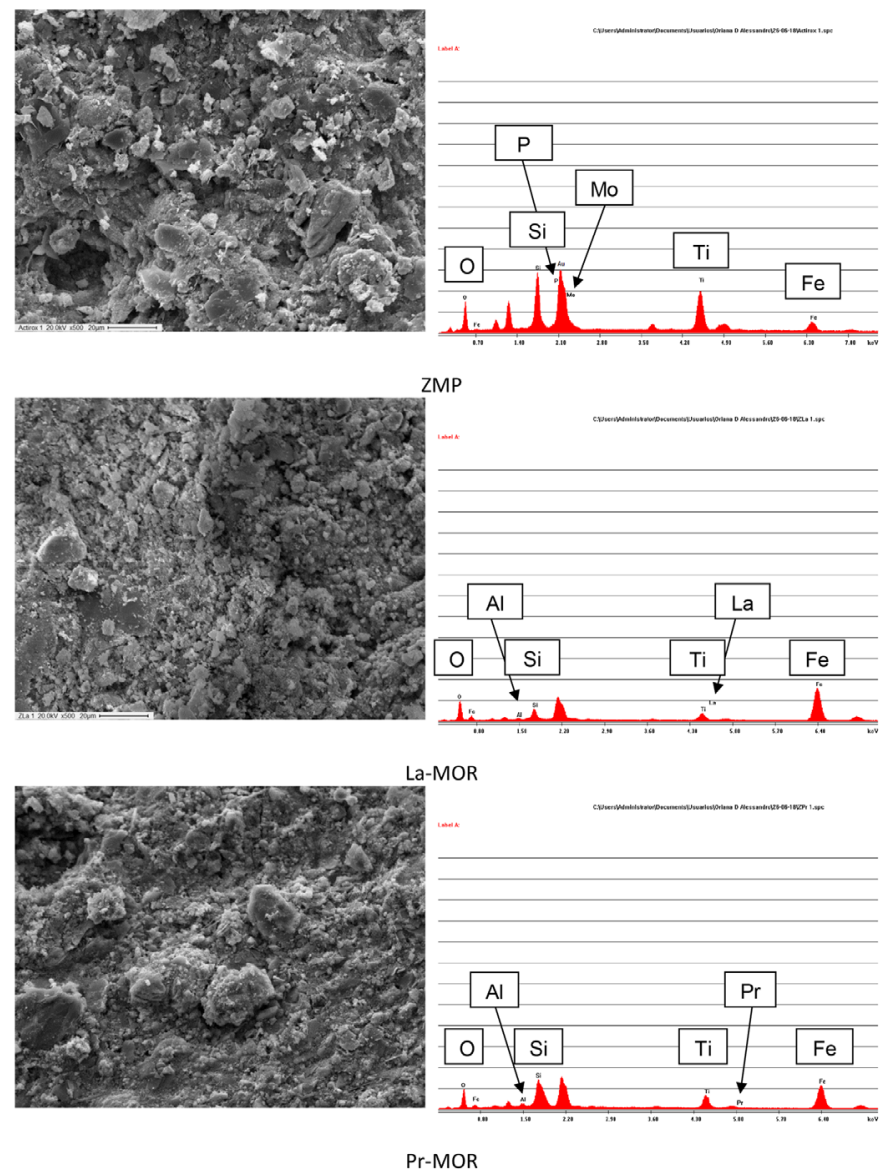

Figura 5. Microfotografías a 500x de los electrodos de trabajo. 
Tabla 2. Cuantificación AED para los electrodos de trabajo.

\begin{tabular}{|l|c|c|c|}
\hline \% peso & ZMP & La-MOR & Pr-MOR \\
\hline $\mathrm{O}$ & 38,77 & 26,94 & 29,77 \\
\hline $\mathrm{Fe}$ & 8,74 & 56,10 & 36,74 \\
\hline $\mathrm{Si}$ & 18,95 & 9,79 & 20,85 \\
\hline $\mathrm{Al}$ & - & 0,89 & 2,14 \\
\hline $\mathrm{La}$ & - & 0,57 & - \\
\hline $\mathrm{Pr}$ & - & - & 0,39 \\
\hline $\mathrm{Ti}$ & 19,33 & 5,71 & 10,11 \\
\hline $\mathrm{Mo}$ & 9,39 & - & - \\
\hline $\mathrm{Zn}$ & 2,26 & - & - \\
\hline $\mathrm{P}$ & 2,56 & - & - \\
\hline Total & 100,00 & 100,00 & 100,00 \\
\hline
\end{tabular}

las cuantificaciones AED para cada uno de los electrodos de trabajo.

La muestra tratada con la pintura que contenía el pigmento anticorrosivo ZMP, revela la presencia de sus componentes característicos Mo, Zn y P, aunque la cuantificación puede acarear error porque los picos de energía del P y del Mo son muy cercanos al del oro, material con el que se realizó el metalizado de todas las muestras.

Las películas protectoras son amorfas, para las muestras con $\mathrm{La}$ y $\mathrm{Pr}$ estaban constituidas principalmente por oxihidróxidos de hierro, lo que indicaría que las pinturas conteniendo zeolitas modificadas resultaron más porosas que la que contenía "molibdofosfato" de zinc. Resultado que coincide con los menores valores de Ri.

La presencia de Ti indica que los componentes de la pintura interactuaron fuertemente con el sustrato de acero formando parte de la película protectora adherente.

Para las muestras pintadas con los productos conteniendo La-MOR y Pr-MOR se encuentra la presencia de $\mathrm{Al}$, indicando que el material zeolítico ha persistido ambos tratamientos, mientras que la presencia de los respectivos cationes de intercambio es escasa, lo que indicaría que los mismos se han lixiviado durante el proceso.

La presencia de $\mathrm{Si}$, en todas las muestras, puede deberse tanto al talco como a la mordenita.

\section{CONCLUSIONES}

Es posible incorporar iones lantano y praseodimio (cationes pasivantes) a la estructura de la mordenita y los sólidos así obtenidos incorporarlos a una pintura alquídica anticorrosiva. Según los resultados de los ensayos electroquímicos, el mejor comportamiento anticorrosivo se logró con el "molibdofosfato" de zinc. La pintura Pr-MOR tuvo un comportamiento intermedio seguido por la pintura La-MOR y por lo tanto podrían ser empleadas en ambientes menos exigentes teniendo en cuenta que con estos nuevos pigmentos que no contienen fosfatos, se evitan los efectos de la eutrofización de los ecosistemas acuáticos.

\section{AGRADECIMIENTOS}

Los autores agradecen al Consejo Nacional de Investigaciones Científicas y Técnicas (CONICET), a la Universidad Nacional de La Plata (UNLP) y a la Comisión de Investigaciones Científicas de Buenos Aires (CICPBA), por el apoyo económico brindado para llevar a cabo esta investigación.

\section{REFERENCIAS}

[1] M.N. Khan andF. Mohammad. "Eutrophication: Causes, Consequences and Control”. Springer. $1^{\text {era }}$ Edición. Vol. 2, pp. 1-15. Londres, Inglaterra. 2014. ISBN: 978-9400778139.

[2] J.M. Yeh, S.J. Liou, C.Y. Lin, C.Y. Cheng, Y.W. Chang and K.R. Lee. "Anticorrosively Enhanced PMMA-Clay Nanocomposite Materials with Quaternary Alkylphosphonium Salt as an Intercalating Agent". Chemistry of Materials. Vol. 14, Issue 1, pp. 154-161. 2002. ISSN: 1520-5002. DOI: $10.1021 / \mathrm{cm} 010337 \mathrm{f}$.

[3] P.A. Wright, K.H.J. Buschow, R.W. Cahn, M.C. Flemings, B. Ilschner, E.J. Kramer, S.Mahajan and P. Veyssière. "Acid Catalysts". Encyclopedia of Materials: Science and Technology. Elsevier. $2^{\text {da }}$ Edición. Vol. 1, pp. 1-6. 2001. Oxford, Inglaterra. ISBN: 978-0-08-043152-9.

[4] D.S. Coomb, A. Alberti, T. Armbuster, G. Artioli, C.E. Colella, J.D. Grice, F. Liebau, J.A. Mandarino, E.H. MinatoNickel, E. Passaglia, D.R. Peacor, S. Quartieri, R. Romano, M. Ross, R.A. Sheppard, E. Tillmanns and G. Vezzalini. "Recommended nomenclarture for zeolite minerals: report of the subcommittee 
on zeolites of the international mineralogical association, commission on new minerals and mineral names". The Canadian Mineralogist. Vol. 35, Issue 6, pp. 1571-1606. 1997. ISSN: 0008-4476.

[5] A. Dyer, K.H.J. Buschow, R.W. Cahn, M.C. Flemings, B. Ilschner, E.J. Kramer, S. Mahajan and P. Veyssière. "Zeolites". Encyclopedia of Materials: Science and Technology. Elsevier. $2^{\text {da }}$ Edición. Vol. 1, pp. 9859-9863. 2001. Oxford, Inglaterra. ISBN: 978-0-08-043152-9.

[6] Z.V.P. Murthy, P.A. Parikh and N.B. Patel. "Application of $\beta$-Zeolite, Zeolite Y, and Mordenite as Adsorbents to Remove Mercury from Aqueous Solutions". Journal of Dispersion Science and Technology. Vol. 34, Issue 6, pp. 747-755. 2013. ISSN: 1532-2351. DOI : $10.1080 / 01932691.2012 .685839$.

[7] V. Córdova-Rodríguez, I. Rodríguez-Iznaga, R.M. Acosta-Chávez, F. Chávez-Rivas, V. Petranovskii and A. Pestryakov. "Use of natural mordenite to remove chromium (III) and to neutralize $\mathrm{pH}$ of alkaline waste waters". Journal of Environmental Science and Health, Part A. Vol. 51, Issue 5, pp. 425-433. 2016. ISSN: 0193-2691. DOI: 10.1080/01932691.2012.685839.

[8] X.S. Wang, J. Huang, H.Q. Hu, J. Wang and Y. Qin. "Determination of kinetic and equilibrium parameters of the batch adsorption of $\mathrm{Ni}(\mathrm{II})$ from aqueous solutions by Na-mordenite". Journal of Hazardous Materials. Vol. 142, Issue 1, pp. 468-476. 2007. ISSN: 0304-3894. DOI: 10.1016/j.jhazmat.2006.08.047.

[9] A. Villarreal, G. Garbarino, P. Riani, E. Finocchio, B. Bosio, J. Ramírez and G. Busca. "Adsorption and separation of $\mathrm{CO}_{2}$ from $\mathrm{N}_{2}$-rich gas on zeolites: $\mathrm{Na}-\mathrm{X}$ faujasite vs Na-mordenite". Journal of $\mathrm{CO}_{2}$ Utilization. Vol. 19, Issue 1, pp. 266-177. 2017. ISSN: 2212-9820. DOI: 10.1016/j.jcou.2017.03.021.

[10] K. Segawa and T. Hiroyasu. "Highly selective methylamine synthesis over modified mordenite catalysts". Journal of Catalysis. Vol. 131, Issue 2, pp. 482-490. 1991. ISSN: 0021-9517. DOI: 10.1016/0021-9517(91)90280-H.

[11] S.M.K. Aboul-Fotouh, N.A.K. Aboul-Gheit and M.M.I. Hassan. "Conversion of Methanol Using Modified H-MOR Zeolite Catalysts". Chinese Journal of Catalysis. Vol. 32,
Issue 3, pp. 412-417. 2011. ISSN:0253-9837. DOI:10.1016/S1872-2067(10)60187-8.

[12] D. Mravec, A. Turan, A. Filková, N. Mikesková, E. Volkovicsová, G. Onyestyák, S. Harnos, F. Lónyi, J. Valyon and A. Kasznyi. "Catalytic etherification of bioglycerol with bioethanol over H-Beta, H-Y and H-MOR zeolites". Fuel Processing Technology. Vol. 159, Issue 1, pp. 111-117. 2017. ISSN:0378-3820. DOI: 10.1016/j.fuproc.2017.01.012.

[13] S. Roselli, N. Bellotti, C. Deyá, M. Revuelta, B. del Amo and R. Romagnoli. "Lanthanumexchanged zeolite and clay as anticorrosive pigments for galvanized steel". Journal of Rare Earths. Vol. 32, Issue 4, pp. 352-359. 2014. ISSN: 1002-0721. DOI: 10.1016/ S1002-0721(14)60078-8.

[14] S. Roselli, C. Deyá, M.V. Revuelta, A. Di Sarli and R. Romagnoli. "Zeolites as reservoirs for $\mathrm{Ce}(\mathrm{III})$ as passivating ions in anticorrosion paints". Corrosion Reviews. Vol. 36, Issue 3, pp. 305-322. 2018. ISSN: 0334-6005. DOI: 10.1515/corrrev-2017-0090.

[15] O. D’Alessandro, G.J. Selmi, C. Deyá, A. Di Sarli and R. Romagnoli. "Formulation and Assessment of a Wash-Primer Containing Lanthanum "Tannate" for Steel Temporary Protection". Journal of Materials Engineering and Performance. Vol. 27, Issue 2, pp. 687704. 2018. ISSN: 1059-9495. DOI: $10.1007 /$ s11665-017-3103-y.

[16] O. D’Alessandro, G.J. Selmi, R. Romagnoli y C. Deyá. "Preparación y caracterización de un material zeolítico intercambiado con $\mathrm{La}^{+3}$ y $\mathrm{Pr}^{+3}$ y estudio de su capacidad como pigmento anticorrosivo". XVII Congreso Internacional de Metalurgia y Materiales. Copiapó, Chile. 2017.

[17] J.N. Murray. "Electrochemical test methods for evaluating organic coatings on metals: An update. Part I Introduction and generalities regarding electrochemical testing of organiccoatings". Progress in Organic Coatings. Vol. 30, Issue 4, pp. 225-233. 1997. ISSN: 0300-9440. DOI: 10.1016/S0300-9440(96)00677-7.

[18] J.H. Leidheiser. "Electrical and electrochemical measurements as predictors of corrosion at the metal-organic coating interface". Progress in Organic Coatings. Vol. 7, Issue 1, pp. 79-104. 1979. ISSN: 0300-9440. DOI: 10.1016/0300-9440(79)80038-7. 